\title{
Ready or Not, AI Comes- An Interview Study of Organizational AI Readiness Factors
}

\author{
Jan Jöhnk $\cdot$ Malte Weißert $\cdot$ Katrin Wyrtki
}

Received: 13 March 2020/Accepted: 20 October 2020/Published online: 22 December 2020

(C) The Author(s) 2020

\begin{abstract}
Artificial intelligence (AI) offers organizations much potential. Considering the manifold application areas, AI's inherent complexity, and new organizational necessities, companies encounter pitfalls when adopting AI. An informed decision regarding an organization's readiness increases the probability of successful AI adoption and is important to successfully leverage AI's business value. Thus, companies need to assess whether their assets, capabilities, and commitment are ready for the individual AI adoption purpose. Research on AI readiness and AI adoption is still in its infancy. Consequently, researchers and practitioners lack guidance on the adoption of AI. The paper presents five categories of AI readiness factors and their illustrative actionable indicators. The AI readiness factors are deduced from an in-depth interview study with $25 \mathrm{AI}$ experts and triangulated with both scientific and practitioner literature. Thus, the paper provides a sound set of organizational AI readiness factors, derives
\end{abstract}

Electronic Supplementary Material The online version of this article (https://doi.org/10.1007/s12599-020-00676-7) contains supplementary material, which is available to authorized users.

Accepted after two revisions by the editors of the special issue

J. Jöhnk $(\bowtie) \cdot$ K. Wyrtki

Project Group Business \& Information Systems Engineering of the Fraunhofer FIT, FIM Research Center, Wittelsbacherring 10, 95444 Bayreuth, Germany

e-mail: jan.joehnk@fit.fraunhofer.de

K. Wyrtki

e-mail: katrin.wyrtki@ fit.fraunhofer.de

M. Weißert

FIM Research Center, University of Bayreuth, Universitätsstraße 30, 95447 Bayreuth, Germany

e-mail: malteweissert@gmail.com corresponding indicators for AI readiness assessments, and discusses the general implications for AI adoption. This is a first step toward conceptualizing relevant organizational AI readiness factors and guiding purposeful decisions in the entire AI adoption process for both research and practice.

Keywords Artificial intelligence $\cdot$ AI adoption $\cdot$ AI readiness · Organizational readiness assessment $\cdot$ Interview study

\section{Introduction}

Artificial intelligence (AI) is a general-purpose technology (GPT) with a unique learning capability that provides organizations with potentials for wide-ranging improvements as well as entirely new business opportunities. Iansiti and Lakhani 2020. Being a GPT, AI drives changes at the task, process, and business model level in a plethora of application areas and as such offers a competitive advantage to organizations (Brynjolfsson and McAfee 2017). Ample access to improved learning algorithms and available AI use cases as well as corresponding solutions facilitate AI adoption in organizations. In 2019, $80 \%$ of large organizations aimed to adopt or had adopted some form of AI (Gartner 2019; Ghosh et al. 2019). However, most organizations use AI in single pilots, whereas only $8 \%$ of organizations have adopted $\mathrm{AI}$ in core practices (Fountaine et al. 2019). Due to AI's nature as a GPT, possible application scenarios are not always directly obvious and organizations must understand the technology to decide on the intended adoption purpose (Jovanovic and Rousseau 2005). Consequently, the adoption of AI poses challenges on an organizational, technical, and individual level (Baier et al. 2019; Bughin et al. 2017). Thus, 
organizations must proactively cope with the challenges caused by AI to mitigate risks and successfully adopt AI.

Research on AI adoption can draw on the two established literature streams of innovation adoption and technology adoption on the organizational level (e.g., Chwelos et al. 2001; Damanpour and Schneider 2006; Hameed et al. 2012). Prior work on innovation adoption has focused on the adoption process (Hameed et al. 2012), different adoption factors (Frambach and Schillewaert 2002), or the application of adoption models to a specific innovation or technology (Oliveira and Martins 2011). Yet, the literature emphasizes there is no unifying, one-size-fits-all theory of innovation adoption (Molla and Licker 2005). Further, besides major general factors, innovation adoption antecedents remain unspecific (Damanpour and Schneider 2006). Organizational readiness for change theory postulates that a higher level of organizational readiness increases the success of innovation adoption and decreases the risk of failure (Snyder-Halpern 2001; Weiner 2009). Mostly, readiness is conceptualized with psychological factors such as the commitment to change, as well as structural constituents as the capability to change and contextual factors (Lokuge et al. 2018; Weiner 2009) without consensus on related relevant factors (Nguyen et al. 2019). Consequently, readiness models require context-specific consideration and need to be tailored to the related domain, i.e. a specific technology (Molla and Licker 2005).

Considering AI's technical characteristics and knowledge barriers, AI adoption implies a high implementation complexity (Gallivan 2001) which differentiates it from other digital technologies that are typically easy-to-use and easy-to-deploy (Lokuge et al. 2018). Hence, AI adoption demands a thorough understanding of relevant AI readiness factors, an operationalized readiness assessment, and a tailored match between an organization's current AI readiness and the aspired AI adoption purpose. Understanding how the concepts of AI readiness and AI adoption relate to each other increases the probability of successful AI adoption and is essential to leverage AI's business value. Although AI adoption draws on research on technology adoption, AI's specifics require a dedicated investigation of readiness factors. Existing investigations on the phenomenon shed light upon the influencing factors of AI adoption with the help of organizational frameworks like TOE (technological, organizational, and environmental) (Alsheibani et al. 2018, 2019; Pumplun et al. 2019). However, it is unclear which factors are relevant for the organizational adoption challenges. We seek to conceptualize AI readiness and important AI readiness factors. This is a prerequisite to enhancing prescriptive knowledge and allowing action-oriented implications for building AI readiness and fostering AI adoption (Alsheibani et al. 2018; Pumplun et al. 2019). Thus, we ask:

What factors constitute organizational AI readiness to guide the AI adoption process?

We conduct an in-depth interview study and collect data from $25 \mathrm{AI}$ experts to conceptualize relevant factors for an organization's AI readiness assessment (Schultze and Avital 2011). We use open and axial coding to deduce AI readiness factors from the interview data (Corbin and Strauss 1990) and further triangulate our conceptualization with readiness and adoption literature as well as insights from existing practitioner studies (Flick et al. 2004). Drawing on our insights, we compile five categories strategic alignment, resources, knowledge, culture, and data - with 18 factors specifying distinct action fields of organizational AI readiness. Further, we operationalize these factors with 58 illustrative indicators and evaluate our results via a card-sorting procedure in an AI-related researchers' focus group. Finally, we discuss the implications for the overarching adoption and readiness concepts as well as for the literature on managing AI.

In sum, our paper is a first step toward comprehensively conceptualizing and operationalizing organizational AI readiness. As such, we provide additional empirical groundwork for theorizing on technology adoption and readiness in general. Further, our AI readiness factors serve as the necessary foundation for purposeful decisions in the entire AI readiness and adoption process. Hence, we extend the body of descriptive knowledge on AI readiness and provide a first building block for prescriptive knowledge to guide organizations toward successful AI adoption. Future research may draw on these results in order to validate our illustrative indicators which assess AI readiness and provide guidance on how to achieve a required AI readiness target level.

\section{Theoretical Background}

This paper's theoretical foundation is twofold: First, with AI being a technological innovation, the literature on innovation adoption provides the scaffold of our research. Second, research on organizational readiness for change emphasizes readiness as a necessary precursor for organizational change, such as AI adoption. In this section, we embed our work on organizational AI readiness in the conceptual frame of adoption and readiness literature.

\subsection{Innovation and Technology Adoption}

Various disciplines (e.g., sociology, psychology, and IS) discuss innovation adoption on an individual and 
organizational level (Gopalakrishnan and Damanpour 1997). Adoption refers to the decision to use an innovation, e.g., product, service, process, technology (Frambach and Schillewaert 2002). To understand the necessities of organizational AI use, we focus on organizational technology adoption. Adoption decisions rest on the expectation of improved organizational performance (Hameed et al. 2012). Hence, the literature investigates the adoption process (Damanpour and Schneider 2006; Rogers 2003), factors that lead to the acquisition and continued use of technology, and related effects on organizational performance (Hameed et al. 2012; Lokuge et al. 2018; Subramanian and Nilakanta 1996).

The established innovation adoption process follows three stages: initiation, adoption decision, implementation (Rogers 2003). In the initiation stage, organizations recognize needs, become aware of innovation, form an attitude toward it, and create a proposal for adoption (Gopalakrishnan and Damanpour 1997). In the adoption decision stage, organizations evaluate an innovation from various perspectives to accept or reject the proposal. In case of acceptance, the implementation stage covers the acquisition of the innovation, the performance of trials as well as continued use (Hameed et al. 2012). In case of rejection, organizations may later choose to reinitiate and reassess the adoption process.

To investigate technology adoption factors on an organizational level, research proposes adoption and implementation theories, e.g., diffusion of innovation theory (DOI) (Rogers 2003), TOE framework (Tornatzky et al. 1990), technology acceptance model (TAM) (Davis 1985), theory of reasoned action (TRA) (Fishbein and Ajzen 1975), or theory of planned behavior (TPB) (Ajzen 1991). For extensive literature reviews see, e.g., Hameed et al. (2012) or Oliveira and Martins (2011). Synthesizing extant theories, four main adoption dimensions exist: Innovation's characteristics (e.g., relative advantage, complexity, compatibility, observability, and trialability), management's commitment to innovation (e.g., CEO characteristics, willingness to change, management support), organizational characteristics (e.g., scope, size, financial resources), and environmental factors (e.g., competitors, suppliers, customers) (Molla and Licker 2005; Sharma and Yetton 2003). However, adoption models must account for the specific technology in focus and its respective context (Molla and Licker 2005). Hence, the research mostly either investigates singular specific adoption factors, e.g., management support (Sharma and Yetton 2003), or provides a compilation of factors based on the above-mentioned theories related to a specific technology and context (Oliveira and Martins 2011).

\subsection{Organizational Readiness for Change}

In line with adoption antecedents, research from different disciplines (e.g., management, health, and IS) discusses the concept of organizational readiness for change (Weiner 2009). In essence, readiness indicates the state necessary to engage in a specific activity, e.g., the adoption of a specific innovation (Lokuge et al. 2018).

In IS, readiness has been discussed in several contexts, e.g., the adoption of technologies (Abdinnour-Helm et al. 2003; Chwelos et al. 2001; Lokuge et al. 2018). In addition to e-readiness, which gained popularity as the degree to which nations or organizations were prepared to benefit from e-innovations (Aboelmaged 2014; Molla and Licker 2005), the literature discusses digital readiness for the adoption of digital technologies (Lokuge et al. 2018; Nguyen et al. 2019). Nguyen et al (2019) define digital readiness as "the degree to which an organization is ready to digitally transform the current organization". As AI classifies as digital technology ( ̊̊gerfalk 2020), we apply digital readiness to understand the precursors of AI adoption. IS literature describes various jumbled readiness factors influencing organizational readiness for technology adoption. For instance, financial and technological resources, management support, organizational culture, commitment, communication of goals, and partnership readiness (Chwelos et al. 2001; Damanpour and Schneider 2006; Iacovou et al. 1995; Lokuge et al. 2018; Robey et al. 2008).

Nguyen et al. (2019) systemize digital readiness according to the three overarching categories of organizational assets, capabilities, and commitment. Both assets and capabilities are part of organizational resources (Helfat and Peteraf 2003). Assets refer to tangible or intangible inputs to production (Helfat and Peteraf 2003), whereas capabilities include organizations' measures to change assets (Nguyen et al. 2019). Commitment describes organizations' willingness and support for innovation initiatives (Molla and Licker 2005; Weiner 2009). Hence, building digital readiness does not describe a one-time transition but focuses on the continuous development of factors in the three categories.

In the past, readiness models have been critiqued regarding several issues. For one thing, readiness models require adaptations to account for organizations' specific context, such as industry or organizational particularities. For another thing, readiness models and their results often bear a bias due to companies' self-assessment. Still, readiness is an important precursor of and organizational capability for successful technology adoption. Also, organizations face the risk of failure when not being ready for adoption. Consequently, readiness models are an important tool to assess the organizational state of preparation to 
exploit the potential of an innovation (Molla and Licker 2005).

\subsection{AI Specifics for Adoption and Readiness}

AI comprises a set of underlying techniques to enable agents to act intelligently (Russell et al. 2016). Hence, organizations may use AI-based systems for various purposes such as autonomous vehicles, medical diagnoses, or virtual assistants (Bughin et al. 2017). AI's broad applicability both to address existing problems as well as to explore entirely new business opportunities allows AI to be characterized as a GPT (Bresnahan and Trajtenberg 1995; Brynjolfsson et al. 2017). In specific, AI's nature as a GPT implies a completely new form of approaching problems of various kinds and enables a significant impact on various industries (Magistretti et al. 2019). Consequently, AI opens a wide-ranging array of different adoption purposes, i.e. organizations' goals and objectives for AI adoption in specific application scenarios. Because of its widespread potential, organizations have to understand AI as technology and derive the right level of ambition for possible applications (Davenport 2018).

Likewise, AI's variety of adoption purposes requires organizations to create the necessary conditions, and introduce managerial practices for successful AI adoption (Brynjolfsson and McAfee 2017; Hofmann et al. 2020). Differing from other digital technologies, AI can hardly be characterized as easy-to-use or easy-to-deploy (Lokuge et al. 2018). Specifically, AI adoption comprises technical (e.g., limited technology capabilities) and non-technical (e.g., lack of leadership support) challenges that arise before and during implementation. Considering these complexities and new organizational necessities, successful AI adoption requires coordinated activities across the organization by fostering their AI readiness first (Alsheibani et al. 2019; Baier et al. 2019; Gallivan 2001). Owing to its specifics and its challenges opposed to other technologies, AI requires an explicit discussion against the backdrop of research on technology adoption and readiness on the organizational level. However, the research on organizational AI adoption and AI readiness is still in its infancy (Alsheibani et al. 2018; Pumplun et al. 2019) (see also Table 2 in Appendix I, available online via http:// springerlink.com).

Alsheibani et al. (2018) describe AI readiness as "the preparedness of organizations to implement change involving applications and technology related to AI". Particularly the AI readiness assessment before the adoption decision enables organizations to proactively identify potentials gaps for successful AI adoption (Alshawi 2007). Such an assessment provides decision-relevant information and reduces uncertainty regarding the AI adoption decision. Further, the continuous assessment of AI readiness enables organizations to steer the development of their assets, capabilities, and commitment (Molla et al. 2009). Consequently, if an organization is capable of accurately measuring its AI readiness and drawing the right conclusions before the adoption decision, it can reduce risk and improve the adoption decision.

Alsheibani et al. (2018) investigate AI readiness through the lens of the TOE framework (Tornatzky et al. 1990). Further, they presume that high AI readiness positively impacts AI adoption success (Alsheibani et al. 2018). Pumplun et al. (2019) also draw on the TOE framework for investigating $\mathrm{AI}$ readiness and extend it with $\mathrm{AI}$-specific factors. However, to the best of our knowledge, there is so far no structured conceptualization of readiness for technology on the organizational perspective. Hence, organizations yet lack guidance on organizational factors, which

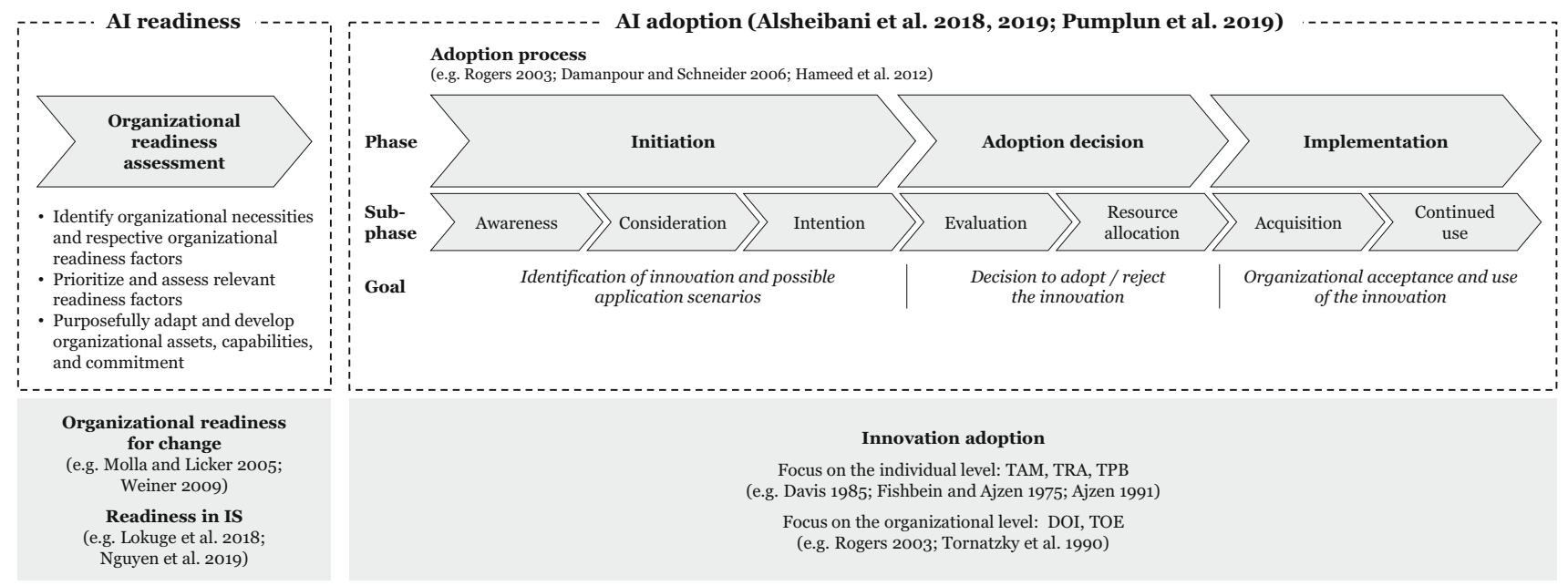

Fig. 1 Our Focus on AI Readiness in the Context of Adoption and Readiness Literature 


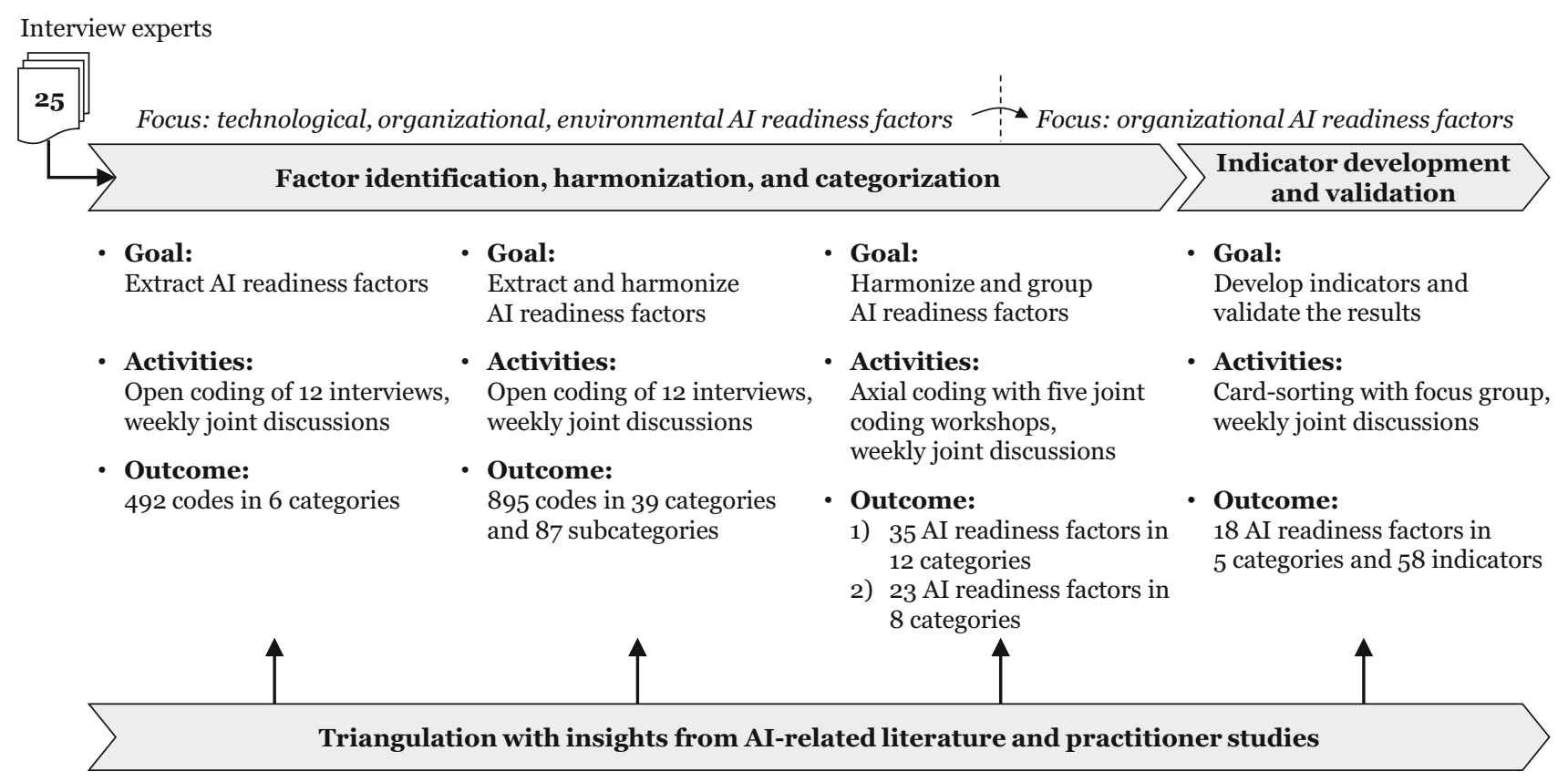

Fig. 2 Overview of Our Research Approach

are necessary to derive action-oriented implications for AI readiness.

Other insights suggest tackling common AI adoption barriers such as a lack of leadership support and a lack of funding (Alsheibani et al. 2019). Yet, avoiding these common pitfalls does not address the underlying infrastructural and cultural preconditions to AI readiness (Pumplun et al. 2019). Further, research remains vague as to where organizations should address these AI-specific factors in the adoption process and what implications arise for the continued use.

In sum, research so far provides fruitful theoretical groundwork but cannot provide relevant organizational AI readiness factors. Drawing on this previous work, we seek to provide a sound set of organizational AI readiness factors and corresponding indicators for AI readiness assessments. This will enable us to better understand AI's specifics and discuss the implications for the AI adoption process as well as the commonalities and distinct features compared to the existing findings for other technologies. Figure 1 summarizes our conceptual foundation and seminal work.

\section{Method}

We used a qualitative research approach to understand AI readiness factors (Bhattacherjee 2012). Thus, we account for the complexity and novelty of the wide-spread AI adoption in companies to derive a thorough understanding of AI readiness factors. We deduced AI readiness factors from both the literature and interviews with $25 \mathrm{AI}$ experts. Figure. 2 summarizes our research approach which we elucidate in the following.

\subsection{Data Collection}

We conducted an in-depth interview study to better understand the challenges and potentials of AI adoption as well as relevant AI readiness factors in companies (Myers and Newman 2007). We used purposive sampling to identify 25 AI experts (E01-E25) from our professional network that are considered as key informants regarding digital transformation and the adoption of emerging technologies such as AI in their organizations (Bhattacherjee 2012) (Bhattacherjee 2012). Our participants contribute perspectives from different organizational contexts (e.g., AI adoption stage, AI users and providers, industry, and company size; see Table 3 in Appendix II). This allowed us to holistically explore AI readiness factors and reflect on their contingencies within the specific organizational context. We stopped data collection after 25 interviews because no significant new topics were brought up and the emerging factors were increasingly repetitive.

We structured the interviews into three parts: First, we asked the participants for their understanding and previous experiences with AI. This created a shared understanding for the rest of the interview (Myers and Newman 2007). Second, we asked for human-AI interaction characteristics and their implications for companies. Third, we asked the 
interviewees for AI-related challenges and potentials, AI readiness factors, and their organizations' efforts toward AI adoption. We asked predominantly open-ended questions to account for our paper's in-depth research approach and to generate rich data (Bhattacherjee 2012; Myers and Newman 2007; Schultze and Avital 2011). We recorded, transcribed, and analyzed 1385 interview minutes with the experts' consent.

\subsection{Data Analysis and Identification of AI Readiness Factors}

For data analysis, we used MAXQDA and started with open coding of 12 interviews. One author assigned descriptive codes to our interviewees' statements to get an understanding of the data's breadth and depth (Saldaña 2013). This first coding round resulted in six broad categories related to AI readiness and a total of 492 codes. Next, the same author continued with a second open coding round for the remaining twelve interviews. In weekly joint sessions, all authors discussed insights and emerging links to align the further coding with the theoretical considerations. After consolidating the coding set, we derived 895 codes in 39 categories and 87 subcategories.

In the third coding round, we used axial coding to specify properties and dimensions of the existing categories and subcategories (Corbin and Strauss 1990). During axial coding, we discussed intermediate results within the research team in five coding workshops to immerse ourselves in the field and gain new insights from the coded data (Saldaña 2013). Each workshop was face-to-face and lasted about $60 \mathrm{~min}$. In these workshops, we reviewed existing codes, discussed ambiguities, and reclassified or renamed factors to enhance clarity and meaning. Thus, we systematically developed AI readiness factors following the established TOE framework and excluded categories, subcategories, and codes with no immediate link to AI readiness. This resulted in 35 AI readiness factors in 12 categories. In line with our research question, we subsequently narrowed the focus on organizational factors in the TOE framework. Thereby, we carved out factors at the locus of companies' managerial actions regarding organizational assets, capabilities, and commitment toward AI. We either transformed technological and environmental factors to depict the organizational measures and reactions to such factors or dropped technological and environmental factors with no organizational representation. This resulted in 23 organizational AI readiness factors in 8 categories. During the entire data analysis, we continuously reflected on the data and our emerging understanding with memoing (Saldaña 2013). Further, we used AI-related literature and practitioner studies to triangulate our conceptualization of AI readiness factors (Flick et al. 2004).
After identifying and consolidating the AI readiness factors, we developed preliminary illustrative indicators that provide a starting point for future research to assess AI readiness (Burton-Jones and Grange 2013). We used feedback from a focus group to further trim and restructure our results (see Appendix III for further details). Next, we present our final compilation of $18 \mathrm{AI}$ readiness factors and 58 illustrative indicators (see Table 5 in Appendix IV for a complete list).

\section{Organizational AI Readiness Factors}

We structure the AI readiness factors in five categories that specify action fields and necessary conditions for successful AI adoption (see Table 1). These categories provide a specific organizational chassis for developing AI readiness (Pumplun et al. 2019). AI characteristics provide further reasoning for each factor's organizational necessity.

\subsection{Strategic Alignment}

AI-business potentials describe an organization's fit and compatibility toward AI innovations (Shahrasbi and Paré 2014). Specifically, AI readiness requires awareness of appropriate use cases for either solving an organizational problem or addressing new opportunities (Hofmann et al. 2020; Pumplun et al. 2019). Additionally, companies must examine AI-based systems for their relative advantage over other solutions (Pumplun et al. 2019). Owing to AI's broad applicability along value chains, organizations need to execute novel approaches for use case discovery that emphasize the opportunity perspective to explore purposeful AI-business potentials (Hofmann et al. 2020).

"In professional life, I would say that there is actually no area in the value chain in which AI cannot be used to fundamentally change something. [...]. Just think of $[\ldots]$ your business cases. Where are things that are not good so far, or are too expensive, or rather need optimization under economic aspects?" (E10)

Customer AI readiness describes the knowledge and acceptance of customers toward using AI-integrated offerings (Pumplun et al. 2019). Owing to AI's inherent complexity, AI-based systems often lack transparency, which hinders customers to accept those offerings. Further, customers who lack knowledge and acceptance build expectations toward AI that cannot be met, leading to frustration when using AI-integrated offerings (Brill et al. 2019). Consequently, organizations need to prepare customers by forming adequate expectations, because customers build acceptance through perceived usefulness (Davis 1985). 
Table 1 Organizational AI Readiness Factors

\begin{tabular}{|c|c|c|c|}
\hline & Factor & AI characteristics & Organizational necessity \\
\hline \multirow[t]{5}{*}{$\begin{array}{l}\text { Strategic } \\
\text { alignment }\end{array}$} & $\begin{array}{l}\text { AI-business } \\
\text { potentials }\end{array}$ & AI functions are highly versatile and broadly applicable & $\begin{array}{l}\text { AI-business potentials ensure that AI adoption is } \\
\text { beneficial and suitable for the organization }\end{array}$ \\
\hline & $\begin{array}{l}\text { Customer AI } \\
\text { readiness }\end{array}$ & $\begin{array}{l}\text { AI use requires an understanding of the complexity and } \\
\text { lack of transparency of learning algorithms }\end{array}$ & $\begin{array}{l}\text { Customer AI readiness enables internal or external } \\
\text { customers to appropriately use AI-integrated } \\
\text { offerings }\end{array}$ \\
\hline & $\begin{array}{l}\text { Top } \\
\text { management } \\
\text { support }\end{array}$ & $\begin{array}{l}\text { AI's inherent complexity poses change not only within but } \\
\text { across organizational levels which requires top } \\
\text { management commitment }\end{array}$ & $\begin{array}{l}\text { Top management support signals AI's strategic } \\
\text { relevance to the organization and fosters AI } \\
\text { initiatives }\end{array}$ \\
\hline & AI-process fit & $\begin{array}{l}\text { AI-based systems are more precise if processes are } \\
\text { structured and provide standardized data input }\end{array}$ & $\begin{array}{l}\text { AI-process fit through standardization, } \\
\text { reengineering, and implementation of new processes } \\
\text { facilitates AI adoption }\end{array}$ \\
\hline & $\begin{array}{l}\text { Data-driven } \\
\text { decision- } \\
\text { making }\end{array}$ & $\begin{array}{l}\text { AI-based systems are fundamentally data-driven and } \\
\text { require openness to incorporate such insights }\end{array}$ & $\begin{array}{l}\text { Data-driven decision-making fosters AI adoption } \\
\text { because both utilize data and statistical methods to } \\
\text { gain insights }\end{array}$ \\
\hline \multirow[t]{3}{*}{ Resources } & $\begin{array}{l}\text { Financial } \\
\text { budget }\end{array}$ & $\begin{array}{l}\text { AI-based systems require high investments to tailor assets } \\
\text { and capabilities to the unique context and data }\end{array}$ & $\begin{array}{l}\text { Strategic allocation of the financial budget for AI } \\
\text { adoption supports the overcoming of initial } \\
\text { obstacles and uncertainty }\end{array}$ \\
\hline & Personnel & $\begin{array}{l}\text { AI adoption requires a broader spectrum of different roles } \\
\text { and know-how for core business use }\end{array}$ & $\begin{array}{l}\text { AI specialists and business analysts with AI know- } \\
\text { how facilitate AI adoption }\end{array}$ \\
\hline & $\begin{array}{l}\text { IT } \\
\text { infrastructure }\end{array}$ & $\begin{array}{l}\text { Deploying AI poses high workloads and data storage } \\
\text { requirements }\end{array}$ & $\begin{array}{l}\text { IT infrastructure enables AI-related activities and AI } \\
\text { integration }\end{array}$ \\
\hline \multirow[t]{3}{*}{ Knowledge } & AI awareness & $\begin{array}{l}\text { AI's underlying concepts, e.g., machine learning or the } \\
\text { autonomy of data-based decision support, are hard to } \\
\text { grasp. }\end{array}$ & $\begin{array}{l}\text { AI awareness ensures that employees have adequate } \\
\text { understanding and expectations toward AI }\end{array}$ \\
\hline & Upskilling & $\begin{array}{l}\text { AI-based systems in core business require every employee } \\
\text { to have a basic understanding of AI }\end{array}$ & $\begin{array}{l}\text { Upskilling enables employees to learn and develop } \\
\text { AI or AI-related skills }\end{array}$ \\
\hline & AI ethics & $\begin{array}{l}\text { AI-based systems are at risk for biased learning and } \\
\text { unethical outcomes }\end{array}$ & $\begin{array}{l}\text { AI ethics comprise measures to prevent bias, safety } \\
\text { violations, or discrimination in AI outcomes }\end{array}$ \\
\hline \multirow[t]{3}{*}{ Culture } & Innovativeness & $\begin{array}{l}\text { Employees' fear of AI-induced job loss threatens } \\
\text { proactive innovativeness }\end{array}$ & $\begin{array}{l}\text { Innovativeness increases employees' willingness to } \\
\text { change the status quo through the application of AI }\end{array}$ \\
\hline & $\begin{array}{l}\text { Collaborative } \\
\text { work }\end{array}$ & $\begin{array}{l}\text { AI deployment relies on integrating different perspectives, } \\
\text { i.e. domain, data, and IT }\end{array}$ & $\begin{array}{l}\text { Collaborative work enables employees to work in } \\
\text { teams and combine different skills }\end{array}$ \\
\hline & $\begin{array}{l}\text { Change } \\
\text { management }\end{array}$ & $\begin{array}{l}\text { Employees' lack of understanding and fear of AI threaten } \\
\text { the acceptance of AI-based systems }\end{array}$ & $\begin{array}{l}\text { Change management helps employees to understand } \\
\text { and cope with AI-induced organizational change }\end{array}$ \\
\hline \multirow[t]{4}{*}{ Data } & $\begin{array}{l}\text { Data } \\
\text { availability }\end{array}$ & $\begin{array}{l}\text { AI-based systems learn through different data types and } \\
\text { large data amounts }\end{array}$ & $\begin{array}{l}\text { Data availability within the organization fuels AI } \\
\text { solutions }\end{array}$ \\
\hline & Data quality & $\begin{array}{l}\text { AI-based systems achieve better results the higher the } \\
\text { quality of the data they learn with }\end{array}$ & Data quality ensures accurate AI outcomes \\
\hline & $\begin{array}{l}\text { Data } \\
\text { accessibility }\end{array}$ & $\begin{array}{l}\text { AI personnel require access to relevant data sources for } \\
\text { deployment }\end{array}$ & $\begin{array}{l}\text { Data accessibility facilitates AI experts to easily } \\
\text { prototype and develop AI solutions }\end{array}$ \\
\hline & Data flow & $\begin{array}{l}\text { Initial and continuous training of AI-based systems } \\
\text { requires smooth and automated data flow }\end{array}$ & $\begin{array}{l}\text { Data flow between its source and its use ensures } \\
\text { high data accessibility to AI experts }\end{array}$ \\
\hline
\end{tabular}

"That is why it ultimately means that the end-user must be prepared, i.e. ideally be taken along, must have a clear expectation or vision of what the (AI) system will be able to do in the first minimal viable product variant. [...]. Let's put it this way: the more it can do and the better it can do what is promised, the higher the acceptance." (E08)

Top management support describes the willingness to commence AI initiatives top-down and to signal support for bottom-up initiatives. Considering the wide range of organizational necessities associated with AI adoption, top management support is crucial for successful AI adoption. Experts stressed an organization can only commit to AI adoption, once the top management provides an organization-wide signal. Strong top management support indicators are integrating AI adoption into strategy (Bughin et al. 2017) and fostering AI knowledge and AI awareness (Baslom and Tong 2019). 
"The use of AI can only work if it [AI adoption] is driven from above and [must] therefore start with the board members." (E10)

AI-process fit describes the necessary linkage between an organization's AI strategy and its processes to increase AI readiness. Changes accompanying AI adoption always affect an organization's processes. In this respect, AI-process fit reflects an organization's compatibility regarding AI and is facilitated by a mature process landscape, i.e. standardized and structured processes (Watson et al. 2019).

“Next, of course, are processes. If I don't have proper processes [...] and if my process allows many individual steps, where a person decides, 'go left, go right...?' So, if a certain degree of standardization is missing, then it is also a very strong hurdle [for AI]." (E24)

Data-driven decision-making (DDDM) is the practice of using insights based on data analytics to make decisions (Catalyst Fund 2020; Provost and Fawcett 2013) instead of relying on "gut feeling or business instinct" (Jarrahi 2018). $D D D M$ not only leads to performance improvements in organizations but also increases AI readiness as it represents the preliminary practice for AI-driven decision making (Colson 2019; Iansiti and Lakhani 2020; Microsoft 2020). Thus, organizations should foster $D D D M$ as an organizational practice and prepare employees for a culture where AI provides complementary insights to support firm decisions (Jarrahi 2018).

"I coach board members who want to turn their division or [...] their company into a data-driven company. And for me, data-driven means above all [...] creating value with data. And, of course, AI is a form of value creation." (E10)

\subsection{Resources}

Financial budget refers to the financial resources that organizations allocate toward AI adoption (Pumplun et al. 2019). Since the AI application lifecycle comprises tailoring AI systems toward an organization's unique context and data, adopting AI is time and cost-intensive (Hummer et al. 2019). Besides, AI adoption requires organizations to invest in building know-how and overcome initial uncertainty about AI capabilities and their value (Alsheibani et al. 2019).

"Because it's just so often that I can't say 'Hey, we're going to start with the topic of AI and make X million budget available and play around a bit and see what comes out of it', that's just not how it works." (E09)
Concerning AI personnel, interviewees have particularly identified business analysts and AI specialists as relevant human resources. Business analysts have an abstract understanding of AI capabilities (Bawack et al. 2019) as well as domain know-how. Therefore, they can facilitate use case discovery and act as translators between business functions and AI specialists (Fountaine et al. 2019). In turn, AI specialists have dedicated AI-related skills (e.g., data scientists) and can develop AI solutions based on self-build and pre-built models (Catalyst Fund 2020).

"So, having people in the organization that are interpreters of the technology, that understand what the business needs [are] and can translate that into technology needs, and vice versa, [...] that whole cycle [...] has to be created." (E03)

The IT infrastructure must be modular to facilitate the integration of new AI applications and have a high capacity for AI-related data-intensive training and testing procedures (Catalyst Fund 2020; Groopman 2018). Thus, organizations focus on developing three underlying IT infrastructure capabilities for AI: data storage capabilities to generate and store large amounts of data; networking capabilities to access, process, and transport data quickly; and scalable computing power capabilities to handle AI workloads (Groopman 2018; Intel 2018).

"Communication technology has come far enough to transport even large amounts of data quickly and we also have hardware that can hold and process this data. [...] And these are the drivers that make it technically possible for us to go beyond what was [previously] possible." (E15)

\subsection{Knowledge}

Employees need AI awareness, i.e. an abstract understanding of cognitive AI functions like perceiving, predicting, or generating (Hofmann et al. 2020). This allows employees to see AI as a versatile tool and its application potential in their specific context or industry. For instance, employees recognize the importance of high-quality input as a prerequisite for high-quality AI outcome (Agrawal et al. 2018). As a consequence of building AI awareness, employees gain adequate expectations toward AI.

"It [creating awareness] simply has to be a continuous process of change, because ideally this awareness is then known throughout the company. People know what you can do with it, what you can't do. And AI should then essentially be a tool [...] to create a solution." (E09) 
Upskilling develops interdisciplinary skillsets that are necessary for the successful implementation of AI projects (Davenport 2018). This comprises equipping employees with AI-related know-how such as statistics, data management, data analytics, or data engineering on the one hand, but also with domain know-how on the other hand (Pumplun et al. 2019). Owing to a shortage of skilled AI specialists in the labor market, it becomes obligatory for organizations to invest in upskilling employees ' capabilities (Davenport 2018; Kruse et al. 2019).

"From the company's point of view, it will be necessary to make upfront investments and train people, train employees, and further qualify them." (E23)

AI ethics includes novel methods to prevent unethical AI outcomes which can be a result of biased learning or input data. In case of not being ethically ready, organizations might blindly rely on biased AI outcomes and be held liable for discrimination even if unintentional (Agrawal et al. 2018; Dattner et al. 2019). For instance, gender bias in data sets for AI hiring tools can result in biased candidate selection (Tambe et al. 2018). To increase AI readiness, organizations need to establish new measures and protocols to prevent discrimination and therefore mitigate risks for liability (Agrawal et al. 2018; Groopman 2018).

"You have to be aware [...] that the data also contains a kind of prejudice or bias. [...]. And if you just stubbornly apply algorithms to any amount of data, then $[. .$.$] decisions will be made but they may not be$ entirely correct." (E15)

\subsection{Culture}

Innovativeness is based on the amount and pace of adaptability that organizational members possess (Kruse et al. 2019). Innovative behavior with a general-purpose technology such as AI requires employees to initiate change at a rapid pace and in many areas so that organizations can realize AIs' full potential. As such, innovative behavior includes experimentation, risk-taking, and diverse problem-solving skills (Microsoft 2020; Yuan and Woodman 2010). Since large organizations tend to rely too much on the status quo (Pumplun et al. 2019), innovative behavior should be particularly encouraged.

"There are simply more innovative employees who are more open-minded [and] find it easier to try out something new. [...]. Who simply playfully face it for once without reservations. Early adopters [...], if you like.” (E12)

Collaborative work describes the degree to which domain experts, AI specialists, and IT departments actively communicate and work together in cross-functional teams (Davenport 2018; Fountaine et al. 2019). In the context of AI, collaborative work is crucial to overcome siloed work and to identify new use cases that are beneficial to the organization (Fountaine et al. 2019). Thus, organizations should promote different forms of collaboration so that employees with different skills can complement each other.

"On the one hand, I'm driving the technology forward, as IT. [...]. On the other hand, I naturally need the business departments, i.e. the users of this technology." (E12)

Change management helps employees to understand and cope with AI-induced organizational change (Pumplun et al. 2019). Particularly, change management is important to reduce misconceptions regarding $\mathrm{AI}$ in terms of fear of possible job loss (Fountaine et al. 2019). This is especially important considering that AI-based systems do not necessarily replace job profiles as a whole, but rather take over repetitive tasks or individual process steps (Brynjolfsson and McAfee 2017). Like customers, employees must be made aware of AI benefits through change management to increase acceptance.

"The challenge then often lies in the dialogue with the employees. Taking them by the hand and reacting to their individual situation. [...] This is a change management task that requires sensitivity." (E24)

\subsection{Data}

Data availability comprises the relevant amount and types of data which are both necessary for AI models to be trained and to generate accurate predictions (Agrawal et al. 2018; Kruse et al. 2019). Regarding data type, interview experts point out that different data types influence AI readiness. Structured data, for instance, which is stored in two-dimensional relational tables (Lin et al. 2018), is easier to utilize for standardized AI applications. On the other hand, unstructured data, such as audio, video, or image files (Groopman 2018), is utilized in more advanced AI applications like object recognition.

"First of all, many people always underestimate the amount of learning data required." (E12)

Data quality specifies different quality dimensions that verify its suitability for use by data consumers. Improving on these quality dimensions increases AI readiness as AImodels need to be trained with high-quality data to generate good results (Davenport 2018; Pumplun et al. 2019). AI-relevant data quality dimensions include, for instance, completeness and correctness (Sidi et al. 2012). Since organizations often face data quality issues with historical 
data, they need to improve on universal capabilities like data preparation, data processing, and data quality assurance to increase data readiness (Groopman 2018; Iansiti and Lakhani 2020; Kruse et al. 2019).

"Even if I have built the biggest or the best machine learning model, if bad data comes in, the result will be bad too. [...] 'Shit in, shit out' is the basic principle behind it." (E08)

Data accessibility includes quick and easy access to data. It is facilitated by access management which grants personnel authorized access to various data sources throughout the organization (Catalyst Fund 2020). Consequently, AI specialists are enabled to prototype and develop AI models with appropriate data (Intel 2018). Furthermore, organizations might simplify data accessibility through data centralization measures (e.g., establish a data lake or a data warehouse) rather than keeping data in distributed data silos (Iansiti and Lakhani 2020; Pumplun et al. 2019).

"There is preparedness [...] in the sense of they have to have ready access to data. That data has to be manageable and manipulatable by these AI systems."

(E03)

Good data flow enables AI specialists to move data from its source to its use. An automated and smooth data flow facilitates the implementation and maintenance of AIbased systems as they continuously process data even after the initial training. Indicators for good data flow include defined extract-transform-load processes, established data pipelines, as well as continuous and automated data streams, among other (Catalyst Fund 2020; Groopman 2018).

"So, on the development side, I am responsible for data pipeline, $[\ldots]$ providing the right data, in the right format, in the right population, in the right quality to our machine learning engineers." (E13)

\subsection{Emerging Insights Beyond the AI Readiness Factors}

The AI readiness categories and factors describe the organizational chassis for developing AI readiness. Besides, our explorative interviews provide insights that help to understand the hurdles for successful AI adoption. Consequently, our findings are a necessary precursor to indicate how organizations can explore AI's potentials. However, owing to AI's characteristics as GPT, AI adoption differentiates from previously discussed technology adoption. Organizations define and pursue individual AI adoption purposes that describe how they seek to accrue value from the wide range of AI's potential application scenarios. Thus, AI adoption can have different facets depending on the distinct adoption purpose. The AI adoption purpose can span from single use-cases to self-contained AI-driven business models. Hence, organizations must understand AI's potentials as of where and how AI can add value.

"There are organizations like Apple and Amazon that are deeply involved with AI, its creation and utilization in a very complex way and getting very significant results from it. So if organizations understood it better and had more resources to explore it they would be capable of generating some fairly significant returns from the investment into AI but most organizations don't really know what's its use, how to get started, what to expect from use cases etc. So, it's still very much an exploratory process for many companies." (E03)

This requires organizations to clearly define their AI adoption purpose. Subsequently, a holistic AI readiness assessment building on our AI readiness factors may help organizations to evaluate their status quo and derive corresponding action fields. However, besides the specific AI adoption purpose that may require a different set of priorities regarding the factors, AI readiness is also contingent on the organizational context (e.g., industry, customers, products).

"It would be good if you had something like a guideline, a checklist, or something where you can determine (a) what degree of maturity the companies have today to be AI-ready and (b) what potential is there specifically in companies for the use of AI. And I think that, from my experience, this is completely dependent on the industry, target customer, product, or whatever." (E19)

Drawing on the 58 self-developed illustrative indicators for the AI readiness factors (see Table 5 in Appendix IV), companies may assess their organizational AI readiness resulting in an overview of their readiness level per factor (see Fig. 4 for an exemplary visualization in Appendix V). While such an assessment bears some potential pitfalls, it also facilitates companies to derive actionable measures to improve their current AI readiness level to the desired target level. This may lead to iterative cycles of (re)defining the AI adoption purpose, assessing and developing AI readiness, and AI adoption.

"If I don't have anything to do with AI in my core business yet, what do I have to do? First, I must find small use cases where I can demonstrate the advantages of the technology. Then I must think about how 
I can make this visible in the organization. Then I must think about how I can build up the competencies. Then I must think about how I can spread this throughout the organization so that it ultimately leads to a continuous change process. These are relatively easy things to write down on paper, but in real life, it looks a bit different.” (E09)

Building on prototypes, experiments, and preceding projects as steps of AI adoption, companies may then push their AI adoption purpose to shift over time. For example, they may opt to start with internal applications before involving the customer interface. Again, this emphasizes AI's specifics as a GPT that offers various opportunities but also necessitates an individual understanding of each application scenario.

"There is my directive that we $[\ldots]$ learn how to use AI. Not at the interface of customers and intermediaries but in internal processes. I would not like to have our learning curve at the expense of our customers or intermediaries if the AI is not yet working well." (E12)

Thus, over time, AI readiness is a recurring issue that must follow the specific AI adoption purpose at hand and consider previous experiences and improvements to the organizational AI readiness level. Therefore, our interviewees describe AI readiness and AI adoption as intertwined concepts that mutually demand and foster each other. In that, AI readiness fosters successful AI adoption (or successful AI adoption necessitates AI readiness) and successful AI adoption fosters AI readiness for future application scenarios (or AI readiness necessitates successful AI adoption in smaller experiences to make AI's potential comprehensible).

"I think people have to experience some kind of epiphany $[\ldots]$. And I believe that these aha experiences $[\ldots]$ is what ultimately makes it so that the acceptance then increases. That means also, in the whole chicken-and-egg principle, is what I develop actually what is accepted?" (E07)

\section{Discussion}

In the following, we will position the results of our exploratory interview study within the existing adoption and readiness literature. Thereby, we conceptualize AI readiness as a valuable addition to the scholarly knowledge base and a necessary foundation for successful AI adoption. Further, we discuss the interdependencies between AI readiness and AI adoption as intertwined concepts. Finally, we summarize the theoretical contributions and practical implications of our work as well as our paper's limitations and promising future research avenues.

\subsection{Conceptualizing Organizational AI Readiness for Successful AI Adoption}

Our interviews emphasize that successful AI adoption requires both understanding and managing AI readiness. Further, drawing on the literature on organizational readiness for change and technology adoption, we argue that AI readiness is a valuable concept to account for the technology and context specifics of readiness (Lokuge et al. 2018). We build on the TOE framework, an established construct of organizational adoption literature, to conceptualize AI readiness from an organizational perspective. AI poses challenges and opportunities that differentiate it from other technologies (see Sect. 2.3 for further details). This emphasizes the need for a specific AI readiness consideration against the backdrop of existing literature on readiness and technology adoption. We aim to present a holistic conceptualization of AI readiness allowing researchers to understand what our results build on and to extend existing knowledge. Considering AI's nature as a GPT, we posit two important perspectives on the AI readiness factors.

First, our results yield new AI-specific readiness factors that emerge from AI's distinct features. This includes, for instance, factors of the category data which is the essential input of AI models, or factors such as AI ethics that emerge from potentially biased AI outcomes (Tambe et al. 2018). Second, our interviews corroborate readiness factors that are comparable to adopting any other technology and are also relevant for AI adoption. For instance, our results confirm the relevance of the well-known adoption factors top management support (Sharma and Yetton 2003) and collaboration (Dewi and Ahamat 2018) for AI adoption. However, although such factors may be almost universally valid, AI requires taking a context-specific perspective on the AI readiness factors. Hence, dependent on the specific context of AI adoption, companies must rearrange their organizational chassis and derive appropriate measures to foster the necessary AI readiness factors. Additionally, we argue that AI implies a purpose-specific consideration owing to AI's nature as a GPT that offers a broad variety of application scenarios. Hence, organizations' adoption purposes may vary over time. Likewise, AI adoption requires a continuous assessment of the AI readiness factors based on the intended adoption purpose. Therefore, AI as a GPT demands a new understanding of the readiness and adoption concept that is both context- and purpose-specific.

Given our results, we conceptualize AI readiness being twofold. First, AI readiness comprises 18 readiness factors along five categories that provide the organizational chassis 
for developing AI readiness. Second, beyond the specific factors, AI readiness entails the understanding of purposeful AI adoption. Thus, conceptualizing AI readiness does not only comprise its constituting factors but also their implications for a purposeful assessment, deriving action fields and suitable measures, and continuous consideration during advancing AI adoption.

However, literature commonly describes adoption and readiness only as two vaguely related concepts. Integrative work to unveil their interdependencies is rare (Molla and Licker 2005). Thus, the readiness concept is often limited to being a sequential precursor to the broader adoption concept (Lippert and Davis 2006). Similarly, the emerging literature on $\mathrm{AI}$ adoption and readiness lacks a thorough discussion of how the two concepts go hand in hand when companies seek to benefit from AI's potentials (Alsheibani et al. 2018; Pumplun et al. 2019). From our interviews, we emphasize the need to establish the necessary prerequisites for AI use in companies. Further, we put forward two important considerations to integrate adoption and readiness literature in the context of AI. First, AI adoption and readiness are distinct yet highly interdependent. Given AI's inherent complexity and the arising new organizational necessities, AI readiness must be an integral part of companies' decisions across the entire adoption process to guide investments, prioritization, and resource allocation (Baier et al. 2019). Consequently, understanding and assessing AI readiness is an important means to purposefully adapt the organizational chassis for successful AI adoption (Pumplun et al. 2019). Second, AI adoption and readiness mutually reinforce (or restrict) one another. AI adoption often follows cycles of exploring and piloting individual use cases, gradually expanding across companies' processes and departments (Hofmann et al. 2020). Thus, AI readiness becomes a permanent yet fluid issue for companies instead of a one-time consideration because AI readiness requirements may vary with the intended use of AI's potentials and experience from prior cycles.

In sum, we conceptualize AI readiness with its 18 factors not as a mere precursor condition but as a foundation and integral element throughout the entire AI adoption process (see Fig. 3). Both concepts foster and necessitate each other which leads to a mutually reinforcing and highly intertwined nature. Hence, with changing context and adoption purposes, AI readiness requires perpetual consideration.

\subsection{Theoretical Contribution and Practical Implications}

Summarizing the results and discussion above, our work empirically conceptualizes AI readiness and its roles for successful AI adoption. The contribution to both the broader literature on technology adoption and readiness as well as the literature on managing $\mathrm{AI}$ in organizations is threefold.

First, our paper provides additional empirical groundwork for theorizing on readiness and adoption. We integrate $\mathrm{AI}$ readiness into the process of $\mathrm{AI}$ adoption and the underlying innovation and technology adoption concepts. We show that readiness is an integral element throughout the entire adoption process instead of a precursor condition (Lippert and Davis 2006). Thus, we conclude that it is not sufficient to establish readiness once before technology adoption because both concepts are highly interdependent and mutually reinforcing. In contrast, the concepts of readiness and adoption need to be discussed in conjunction but as distinct concepts that each offers a valuable lens on (intended) technology use in organizations. So far, literature has failed to take such an integrative stance. We understand our work as a step in this direction to better guide managerial actions for organizational readiness that unfold their full potential for successful technology adoption.

Second, we contribute to the emerging literature on AI readiness and AI adoption (Alsheibani et al. 2018; Pumplun et al. 2019). Based on AI's underlying technological characteristics and the arising organizational necessities for successful AI adoption, we derived 18 AI readiness factors in five categories that conceptualize AI readiness. With illustrative indicators, we provide a starting point for distinct action fields for developing AI readiness. In doing so, we corroborate existing factors but also provide additional factors accounting for AI's specifics. We posit that AI poses different opportunities and challenges compared to other technologies owing to its nature as GPT. Thus, AI requires a more thorough and integrated understanding of readiness and adoption because it offers a broad variety of application scenarios. Thus, we argue that while we draw on our existing understanding of the two concepts, AI readiness requires both context- and purpose-specific consideration. The holistic nature of our categories, factors, and illustrative indicators may also serve as a blueprint for deriving relevant organizational readiness factors for other (digital) technologies and elaborating on the interdependencies between their respective adoption purpose and success (Lokuge et al. 2018).

Third, we contribute to the literature on managing AI in organizations (Brynjolfsson and Mitchell 2017). Companies face several difficulties during AI adoption, for instance, the identification of suitable use cases to utilize AI's potentials (Hofmann et al. 2020). By conceptualizing organizational AI readiness, we provide relevant factors and indicators to better understand and assess what measures organizations require for successful AI adoption. Further, we argue that assessing AI readiness exposes action fields for AI adoption stemming from both AI's 


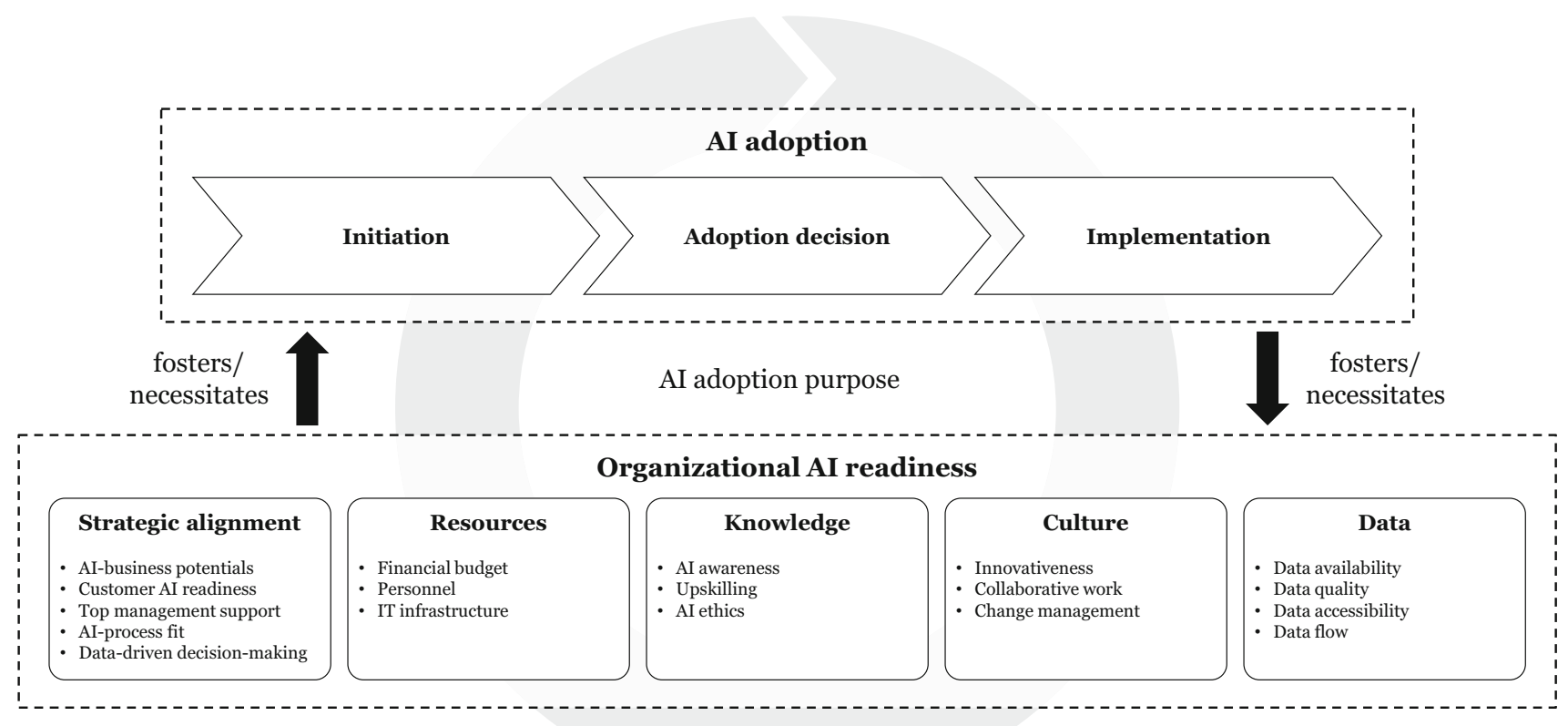

Fig. 3 Integrating AI Readiness in the AI Adoption Process

context and purpose specifics. Thus, research into AI readiness can help to alleviate the difficulties with AI adoption and propose suitable adaptations to the organizational chassis as prescriptive guidance. Thus, our results serve as the descriptive groundwork for further research on AI management in organizations, organizational readiness, and technology adoption.

Regarding practical implications, our paper grants insights into opportunities and challenges for AI adoption. The AI readiness factors provide comprehensive guidance to decision-makers on relevant managerial action fields. Based on an AI readiness assessment, decision-makers may reflect and adapt the factors to specific organizational needs. Setting and developing adequate AI readiness target levels is compulsory in order to derive actionable measures for successful AI adoption. Thus, our results assist organizations in managing the AI adoption process and guide AI investments, resource allocation, and prioritization.

\subsection{Limitations and Future Research}

Our results are subject to limitations that stimulate further research. According to our purposive cross-industry interview sample, our results primarily reflect the perspectives of experts in management positions. Further research may address this limitation by two different means: First, sampling across various job levels could broaden the perceptions and judgments on AI readiness. Second, we propose to further explore organizations' specifics for AI adoption, for instance, through in-depth case studies. This could also help to differentiate AI readiness factors depending on the organizational context and specific AI adoption purpose (Bughin et al. 2017; Pumplun et al. 2019).

Further, we provided insights into the complex interrelations of AI readiness based on our comprehensive understanding and discussion of AI readiness factors. However, we do not elucidate the factors' and indicators' prioritization and weighting concerning the overarching AI adoption purpose and specific organizational contingencies. Thus, future work may continue to validate the factors and our self-developed illustrative indicators in a first step to explore how individual factors and their combinations influence AI adoption success in the subsequent step. A quantitative research approach similar to our exemplary AI readiness assessment seems promising to further validate our results (see similar work from other domains, e.g., Lokuge et al. 2018; Yang et al. 2015).

Finally, our findings explicate the need for contextspecific considerations for organizational readiness by referring to AI's characteristics. While we propose AI readiness factors, we also acknowledge that synergies concerning the readiness for other (digital) technologies may exist. A comparison of organizational readiness factors for several technologies based on their underlying technological features lies beyond the scope of our paper. Such research could add to a more comprehensive understanding of organizational necessities as companies usually strive to adopt and combine multiple (digital) technologies. 


\section{Conclusion}

This paper provided a thorough conceptualization of organizational AI readiness. We combined insights from interviews with $25 \mathrm{AI}$ experts with findings from scientific and practitioner literature to compile 18 AI readiness factors and 58 illustrative indicators in five categories. Further, we discussed that organizations must continuously assess and develop their AI readiness in the AI adoption process and described relevant aspects to consider. This includes AI's nature as a GPT, the context- and purposespecifics, and the mutually reinforcing interplay of AI readiness and AI adoption. Future research should validate our findings and examine the impacts of contingencies and prioritizing AI readiness factors on AI adoption success.

Acknowledgments This research was (in part) carried out in the context of the Project Group Business \& Information Systems Engineering of the Fraunhofer FIT.

Funding Open Access funding enabled and organized by Projekt DEAL.

Open Access This article is licensed under a Creative Commons Attribution 4.0 International License, which permits use, sharing, adaptation, distribution and reproduction in any medium or format, as long as you give appropriate credit to the original author(s) and the source, provide a link to the Creative Commons licence, and indicate if changes were made. The images or other third party material in this article are included in the article's Creative Commons licence, unless indicated otherwise in a credit line to the material. If material is not included in the article's Creative Commons licence and your intended use is not permitted by statutory regulation or exceeds the permitted use, you will need to obtain permission directly from the copyright holder. To view a copy of this licence, visit http://creativecommons. org/licenses/by/4.0/.

\section{References}

Abdinnour-Helm S, Lengnick-Hall ML, Lengnick-Hall CA (2003) Pre-implementation attitudes and organizational readiness for implementing an enterprise resource planning system. Euro $\mathrm{J}$ Oper Res 146:258-273

Aboelmaged MG (2014) Predicting e-readiness at firm-level: an analysis of technological, organizational and environmental (TOE) effects on e-maintenance readiness in manufacturing firms. Int J Inf Manag 34:639-651

Ågerfalk PJ (2020) Artificial intelligence as digital agency. Euro J Inf Syst 29:1-8

Agrawal A, Gans J, Goldfarb A (2018) Prediction machines: the simple economics of artificial intelligence. Harvard Business Review Press, Boston

Ajzen I (1991) The theory of planned behavior. Organ Behav Hum Decis Proc 50:179-211

Alshawi M (2007) Rethinking IT in construction and engineering. Routledge, London

Alsheibani S, Cheung Y, Messom C (2018) Artificial intelligence adoption: AI-readiness at firm-level. In: PACIS 2018 Proceedings, Yokohama
Alsheibani SA, Cheung Y, Messom C (2019) Factors inhibiting the adoption of artificial intelligence at organizational-level: a preliminary investigation. In: AMCIS 2019 Proceedings, Cancún

Baier L, Jöhren F, Seebacher S (2019) Challenges in the deployment and operation of machine learning in practice. In: ECIS 2019 Proceedings, Stockholm-Uppsala

Baslom MMM, Tong S (2019) Strategic management of organizational knowledge and employee's awareness about artificial intelligence with mediating effect of learning climate. Int $\mathbf{J}$ Comput Intell Syst 12:1585-1591

Bawack R, Wamba S, Carillo K (2019) Artificial intelligence in practice: implications for information systems research. In: AMCIS 2019 Proceedings, Cancún

Bhattacherjee A (2012) Social science research: principles, methods, and practices, 2nd edn. CreateSpace Independent Publishing Platform, North Charleston

Boateng GO, Neilands TB, Frongillo EA, Melgar-Quiñonez HR, Young SL (2018) Best practices for developing and validating scales for health, social, and behavioral research: a primer. Front Public Health 6:149-167

Bresnahan TF, Trajtenberg M (1995) General purpose technologies 'engines of growth'? J Econom 65:83-108

Brill TM, Munoz L, Miller RJ (2019) Siri, Alexa, and other digital assistants: a study of customer satisfaction with artificial intelligence applications. J Mark Manag 35:1401-1436

Brynjolfsson E, McAfee A (2017) The business of artificial intelligence: What it can - and cannot - do for your organization. Harvard business review. https://starlab-alliance.com/wp-con tent/uploads/2017/09/The-Business-of-Artificial-Intelligence. pdf. Accessed 12 Mar 2020

Brynjolfsson E, Mitchell T (2017) What can machine learning do? Workforce implications. Science 358:1530-1534

Bughin J, Hazan E, Ramaswamy S, Henke N, Trench M, Dahlström P, Allas T, Chui M (2017) Artificial intelligence: the next digital frontier. https://www.mckinsey.com/ /media/McKinsey/Indus tries/Advanced\%20Electronics/Our\%20Insights/How\%20artifi cial $\% 20$ intelligence $\% 20$ can $\% 20$ deliver $\% 20$ real $\% 20$ value $\%$ 20to\%20companies/MGI-Artificial-Intelligence-Discussionpaper.ashx. Accessed 23 Feb 2020

Burton-Jones A, Grange C (2013) From use to effective use: a representation theory perspective. Inf Syst Res 24:632-658

Catalyst Fund (2020) Get started - AI readiness toolkit. https://www. techstartups101.com/get-started-ai-readiness-toolkit. Accessed $23 \mathrm{Feb} 2020$

Chwelos P, Benbasat I, Dexter AS (2001) Research report: empirical test of an EDI adoption model. Inf Syst Res 12:304-321

Colson E (2019) What AI-driven decision making looks like. https:// hbr.org/2019/07/what-ai-driven-decision-making-looks-like. Accessed 23 Feb 2020

Corbin JM, Strauss A (1990) Grounded theory research: procedures, canons, and evaluative criteria. Qual Sociol 13:3-21

Damanpour F, Schneider M (2006) Phases of the adoption of innovation in organizations: effects of environment, organization and top managers. Br J Manag 17:215-236

Dattner B, Chamorro-Premuzic T, Buchband R, Schettler L (2019) The legal and ethical implications of using AI in hiring. https:// hbr.org/2019/04/the-legal-and-ethical-implications-of-using-aiin-hiring. Accessed 23 Feb 2020

Davenport T (2018) From analytics to artificial intelligence. J Bus Anal 1:73-80

Davis F (1985) A technology acceptance model for empirically testing new end-user information systems: theory and results. Sloan School of Management, Massachusetts Institute of Technology, Massachusetts

DeVellis RF (2017) Scale development: theory and applications, 4th edn. Sage, Thousand Oaks 
Dewi S, Ahamat A (2018) The role of entrepreneurial orientation in achieving organization performance through business model innovation and asset relational collaboration. Hum Soc Sci Rev 6:100-105

Fishbein M, Ajzen I (1975) Belief, attitude, intention and behavior: an introduction to theory and research. Addison-Wesley series in social psychology. Addison-Wesley, Reading, Boston

Flick U, von Kardorff E, Steinke I (2004) A companion to qualitative research. Sage, London

Fountaine T, McCarthy B, Saleh T (2019) Building the AI-powered organization. Harv Bus Rev 97:62-73

Frambach RT, Schillewaert N (2002) Organizational innovation adoption: a multi-level framework of determinants and opportunities for future research. J Bus Res 55:163-176

Gallivan MJ (2001) Organizational adoption and assimilation of complex technological innovations. SIGMIS Database 32:51

Gartner (2019) CIO agenda survey. https://www.gartner.com/smarter withgartner/3-barriers-to-ai-adoption/. Accessed 26 June 2020

Ghosh B, Daugherty PR, Wilson HJ, Burden A (2019) Taking a systems approach to adopting AI. Harv Bus Rev May

Gopalakrishnan S, Damanpour F (1997) A review of innovation research in economics, sociology and technology management. Omega 25:15-28

Groopman J (2018) AI readiness: five areas businesses must prepare for success in artificial intelligence. https://www.kaleidoinsights. com/order-reports/artificial-intelligence-ai-readiness/. Accessed 23 Feb 2020

Hameed MA, Counsell S, Swift S (2012) A conceptual model for the process of IT innovation adoption in organizations. J Eng Technol Manag 29:358-390

Helfat CE, Peteraf MA (2003) The dynamic resource-based view: capability lifecycles. Strateg Manag J 24:997-1010

Hofmann P, Jöhnk J, Protschky D, Urbach N (2020) Developing purposeful AI use cases - a structured method and its application in project management. In: Wirtschaftsinformatik 2020 Proceedings, Potsdam

Hummer W, Muthusamy V, Rausch T, Dube P, El Maghraoui K, Murthi A, Oum P (2019) ModelOps: cloud-based lifecycle management for reliable and trusted AI. In: IC2E 2019 Proceedings, Prague

Iacovou CL, Benbasat I, Dexter AS (1995) electronic data interchange and small organizations: adoption and impact of technology. MIS Q 19:465

Iansiti M, Lakhani KR (2020) Competing in the age of AI. Harv Bus Rev January-February:60-67

Intel (2018) The AI readiness model: judging an organization's ability to generate business value from artificial intelligence. https:// www.intel.com/content/dam/www/public/us/en/documents/ white-papers/ai-readiness-model-whitepaper.pdf. Accessed 23 Feb 2020

Jarrahi MH (2018) Artificial intelligence and the future of work: Human-AI symbiosis in organizational decision making. Bus Horiz 61:577-586

Jovanovic B, Rousseau PL (2005) General purpose technologies. vol 1. Elsevier, Amsterdam, pp 1181-1224

Kruse L, Wunderlich N, Beck R (2019) Artificial intelligence for the financial services industry: what challenges organizations to succeed. In: HICSS 2019 Proceedings

Lin Y, Jun Z, Hongyan M, Zhongwei Z, Zhanfang F (2018) A method of extracting the semi-structured data implication rules. Procedia Comp Sci 131:706-716

Lippert SK, Davis M (2006) A conceptual model integrating trust into planned change activities to enhance technology adoption behavior. J Inf Sci 32:434-448
Lokuge S, Sedera D, Grover V, Dongming X (2018) Organizational readiness for digital innovation: development and empirical calibration of a construct. Inf Manag 56:445-461

Magistretti S, Dell'Era C, Messeni Petruzzelli A (2019) How intelligent is Watson? enabling digital transformation through artificial intelligence. Bus Horiz 62:819-829

Microsoft (2020) Determine how ready your business is for AI. https://info.microsoft.com/ww-landing-ai-maturity-model-web site.html?lcid=en-us. Accessed 23 Feb 2020

Molla A, Licker PS (2005) Perceived e-readiness factors in e-commerce adoption: an empirical investigation in a developing country. Int J Electron Commer 10(1):83-110

Molla A, Cooper V, Pittayachawan S (2009) IT and eco-sustainability: developing and validating a green IT readiness model. In: ICIS 2009 Proceedings, Phoenix

Moore GC, Benbasat I (1991) Development of an instrument to measure the perceptions of adopting an information technology innovation. Inf Syst Res 2:192-222

Myers MD, Newman M (2007) The qualitative interview in IS research: examining the craft. Inf Organ 17:2-26

Nguyen DK, Broekhuizen T, Dong JQ, Verhoef P (2019) Digital readiness: construct development and empirical validation. In: ICIS 2019 Proceedings, Munich

Oliveira T, Martins M (2011) Literature review of information technology adoption models at firm level. EJISE 14:110-121

PA Office of Administration (2018) Artificial intelligence assessment tool. https://www.oa.pa.gov/Policies/Documents/rfd_bus012a. xlsx. Accessed 23 Feb 2020

Pringle T, Zoller E (2018) How to achieve AI maturity and why it matters: an AI maturity assessment model and road map for CSPs. https://www.amdocs.com/sites/default/files/filefield_ paths/ai-maturity-model-whitepaper.pdf. Accessed 23 Feb 2020

Provost F, Fawcett T (2013) Data science and its relationship to big data and data-driven decision making. Big Data 1:51-59

Pumplun L, Tauchert C, and Heidt M (2019) A new organizational chassis for artificial intelligence - exploring organizational readiness factors. In: ECIS 2019 Proceedings, StockholmUppsala

Robey D, Im G, Wareham J (2008) Theoretical foundations of empirical research on interorganizational systems: assessing past contributions and guiding future directions. JAIS 9:497-518

Rogers EM (2003) Diffusion of innovations, 5th edn. Free Press, New York

Russell SJ, Norvig P, Davis E, Edwards D (2016) Artificial intelligence: a modern approach, 3rd edn. Pearson, Edinburgh

Saldaña J (2013) The coding manual for qualitative researchers, 2nd edn. Sage, Los Angeles

Schultze U, Avital M (2011) Designing interviews to generate rich data for information systems research. Inf Organ 21:1-16

Shahrasbi N, Paré G (2014) Rethinking the concept of organizational readiness: what can is researchers learn from the change management field? In: AMCIS 2014 Proceedings, Savannah

Sharma Y (2003) The contingent effects of management support and task interdependence on successful information systems implementation. MIS Q 27:533

Sidi F, Shariat Panahy PH, Affendey LS, Jabar MA, Ibrahim H, Mustapha A (2012) Data quality: a survey of data quality dimensions. In: CAMP'12, Kuala Lumpur

Snyder-Halpern R (2001) Indicators of organizational readiness for clinical information technology/systems innovation: a Delphi study. Int J Med Inform 63:179-204

Subramanian A, Nilakanta S (1996) Organizational innovativeness: exploring the relationship between organizational determinants of innovation, types of innovations, and measures of organizational performance. Omega 24:631-647 
Tambe P, Cappelli P, Yakubovich V (2018) Artificial intelligence in human resources management: challenges and a path forward. Calif Manag Rev 61:15-42

Tornatzky LG, Fleischer M, Chakrabarti AK (eds) (1990) The processes of technological innovation, 4th edn. Lexington Books, Lexington

Watson J, Hatfield S, Wright D, Howard M, Witherick D, Coe L, Horton R (2019) Automation with intelligence: reimagining the organisation in the 'age of with'. https://www2.deloitte.com/ content/dam/Deloitte/tw/Documents/strategy/tw-Automationwith-intelligence.pdf. Accessed 8 Mar 2020
Weiner BJ (2009) A theory of organizational readiness for change. Implement Sci 4:1-9

Yang Z, Sun J, Zhang Y, Wang Y (2015) Understanding SaaS adoption from the perspective of organizational users: a tripod readiness model. Comput Hum Behav 45:254-264

Yuan F, Woodman RW (2010) Innovative behavior in the workplace: the role of performance and image outcome expectations. Acad Manag J 53:323-342 